\title{
Optimization Methods in Computational Fluid Dynamics
}

\author{
Antony Jameson and, assisted by, Kui Ou \\ Aeronautics and Astronautics Department, Stanford University, Stanford, CA, USA
}

1 Introduction: Aerodynamic Design

2 Aerodynamic Optimization and Control Theory

3 Formulation of the Design Problem as a Control Problem

4 Design Optimization Procedure

5 Airfoil Design for Potential Flow using Conformal Mapping

6 Wing Design using the Euler Equations

7 Design Case Studies

8 Conclusion

Acknowledgment

References

\section{INTRODUCTION: AERODYNAMIC DESIGN}

In order to set the context for aerodynamic shape optimization, the airplane design process is summarized in the following paragraphs. It can generally be divided into three phases: conceptual design, preliminary design, and final detailed design, as illustrated in Figure 1. The conceptual design stage defines the mission in the light of anticipated market requirements and determines a general preliminary configuration capable of performing this mission, together

Encyclopedia of Aerospace Engineering. Edited by Richard Blockley and Wei Shyy

(c) 2010 John Wiley \& Sons, Ltd. ISBN: 978-0-470-68665-2
7

9 12 13

with first estimates of size, weight, and performance. In the preliminary design stage, the aerodynamic shape and structural skeleton progress to the point where detailed performance estimates can be made and guaranteed to potential customers. At this stage, the development costs are still fairly moderate, in the range of 50-100 million dollars. In the final design stage, the structure must be defined in complete detail, together with complete systems, including the flight deck, 


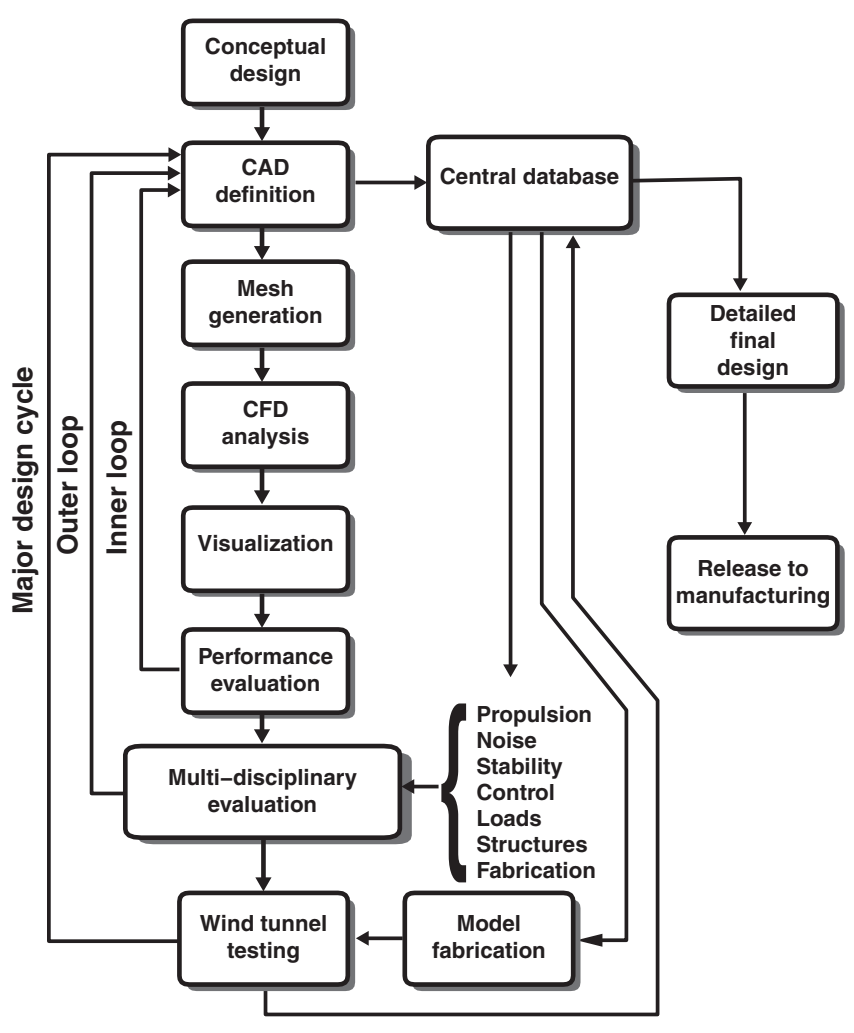

Figure 2. The aerodynamic design process.

control systems (involving major software development for fly-by-wire systems), electrical and hydraulic systems, landing gear, weapon systems for military aircraft, and cabin layout for commercial aircraft. Major costs are incurred at this stage, during which it is also necessary to prepare a detailed manufacturing plan, together with appropriate facilities and tooling. The development costs to reach the point of initial production are in the range of 3-10 billion dollars. Thus, the final design would normally be carried out only if sufficient orders have been received to indicate a reasonably high probability of recovering the investment.

In the development of commercial aircraft, aerodynamic design plays a leading role in the preliminary design stage. The definition of the external aerodynamic shape may actually be finalized in the preliminary design. The aerodynamic lines of the Boeing 777 were frozen, for example, when initial orders were accepted before the initiation of the detailed design of the structure. Figure 2 illustrates the way in which the aerodynamic design process is embedded in the overall preliminary design. The starting point is an initial Computer Aided Design (CAD) definition resulting from the conceptual design. The inner loop of aerodynamic analysis is contained in an outer multi-disciplinary loop, which is in turn contained in a major design cycle involving wind tunnel testing. In recent Boeing practice, three major design cycles, each requiring about 4-6 months, have been used to finalize the wing design.

\section{AERODYNAMIC OPTIMIZATION AND CONTROL THEORY}

The use of computational simulation to scan many alternative designs has proved extremely valuable in practice, but it is also evident that the number of possible design variations is too large to permit their exhaustive evaluation, and thus it is very unlikely that a truly optimum solution can be found without the assistance of automatic optimization procedures. To ensure the realization of the true best design, the ultimate goal of computational simulation methods should not just be the analysis of prescribed shapes but the automatic determination of the true optimum shape for the intended application.

The need to find optimum aerodynamic designs was already well recognized by the pioneers of classical aerodynamic theory. A notable example is the determination that the optimum span-load distribution that minimizes the induced drag of a monoplane wing is elliptic (Glauert, 1926; Prandtl and Tietjens, 1934). There are also a number of famous results for linearized supersonic flow. The body of revolution of minimum drag was determined by Sears (1947), while conditions for minimum drag of thin wings due to thickness and sweep were derived by Jones (1981). The problem of designing a two-dimensional profile to attain a desired pressure distribution was studied by Lighthill (1945), who solved it for the case of incompressible flow with a conformal mapping of the profile to a unit circle. The speed over the profile is

$$
q=\frac{1}{h}|\nabla \phi|
$$

where $\phi$ is the potential that is known for incompressible flow and $h$ is the modulus of the mapping function. The surface value of $h$ can be obtained by setting $q=q_{\mathrm{d}}$, where $q_{\mathrm{d}}$ is the desired speed, and since the mapping function is analytic, it is uniquely determined by the value of $h$ on the boundary. A solution exists for a given speed $q_{\infty}$ at infinity only if

$$
\frac{1}{2 \pi} \oint q \mathrm{~d} \theta=q_{\infty}
$$

and there are additional constraints on $q$ if the profile is required to be closed.

These classical results were limited to special cases amenable to analytic methods and could not readily be 
extended to realistic configurations. This is the underlying motivation for the combination of computational fluid dynamics with numerical optimization methods. Some of the earliest studies of such an approach were made by Hicks and Henne (Hicks, Murman and Vanderplaats, 1974; Hicks and Henne, 1979). The principal obstacle was the large computational cost of determining the sensitivity of the cost function to variations of the design parameters by repeated calculation of the flow. Another way to approach the problem is to formulate aerodynamic shape design within the framework of the mathematical theory for the control of systems governed by partial differential equations (Lions, 1971). In this view, the wing is regarded as a device to produce lift by controlling the flow, and its design is regarded as a problem in the optimal control of the flow equations by changing the shape of the boundary. If the boundary shape is regarded as arbitrary within some requirements of smoothness then the full generality of shapes cannot be defined with a finite number of parameters, and one must use the concept of the Frechet derivative of the cost with respect to a function. Clearly, such a derivative cannot be determined directly by separate variation of each design parameter, because there are now an infinite number of these.

Using techniques of control theory, however, the gradient can be determined indirectly by solving an adjoint equation. Once the gradient has been calculated, a descent method can be used to determine a shape change that will make an improvement in the design. The gradient can then be recalculated, and the whole process can be repeated until the design converges to an optimum solution, usually within $50-100$ cycles. The fast calculation of the gradients makes optimization computationally feasible even for designs in three-dimensional viscous flow. There is a possibility that the descent method could converge to a local minimum rather than the global optimum solution. In practice, this has not proved a difficulty, provided care is taken in the choice of a cost function that properly reflects the design requirements.

Conceptually, with this approach, the problem is viewed as infinitely dimensional, with the control being the shape of the bounding surface. Eventually, the equations must be discretized for a numerical implementation of the method. For this purpose, the flow and adjoint equations may either be separately discretized from their representations as differential equations or, alternatively, the flow equations may be discretized first and the discrete adjoint equations then derived directly from the discrete flow equations. These two approaches are generally called the continuous and discrete adjoint methods, respectively. Both have proved quite successful in practice.

The first application of control theory to shape optimization was by Pironneau (1984), who studied the use of control theory for optimal shape design of systems governed by elliptic equations in the context of finite element methods. Concepts from control theory were first applied to shape optimization in transonic flow by Jameson (1988), who derived the adjoint equations for transonic potential flow and flow governed by the Euler equations. Early numerical results were given in the studies by Jameson (1989), Reuther and Jameson (1995), and Jameson (1995). The adjoint equations have also been used by Baysal and Eleshaky (1991) and by Ta' asan, Kuruvila and Salas (1992), who have implemented a one-shot approach in which the constraint represented by the flow equations is only required to be satisfied by the final converged solution. In their work, computational costs are also reduced by applying multigrid techniques to the geometry modifications as well as the solution of the flow and adjoint equations. Adjoint methods have been applied to incompressible viscous flow problems by Cabuk, Shung and Modi (1991), Huan and Modi (1994), and Desai and Ito (1994). Early applications of adjoint methods on unstructured meshes include the work of Elliot and Peraire (1996), Anderson and Venkatakrishnan (1997), and Nielsen and Anderson (1999).

Recently, there has been a growing interest in evolutionary algorithms that do not make use of the gradient (Gonzalez et al., 2004; LeDoux, et al., 2004; Epstein and Peigin, 2005). While they incur extremely high computational costs, these algorithms have the advantage that they can avoid being trapped in a local minimum. The present chapter is focused on gradient-based methods of proven efficiency. Ultimately, however, one can anticipate that gradient-based methods will be incorporated inside evolutionary methods to combine the advantages of both.

\section{FORMULATION OF THE DESIGN PROBLEM AS A CONTROL PROBLEM}

The simplest approach to optimization is to define the geometry through a set of design parameters, which may, for example, be the weights $\alpha_{i}$ applied to a set of shape functions $b_{i}(x)$ so that the shape is represented as

$$
f(x)=\sum \alpha_{i} b_{i}(x)
$$

Then, a cost function $I$ is selected, which might, for example, be the drag coefficient or the lift-to-drag ratio, and $I$ is regarded as a function of the parameters $\alpha_{i}$. The sensitivities $\partial I / \partial \alpha_{i}$ may now be estimated by making a small variation $\delta \alpha_{i}$ in each design parameter in turn and recalculating the flow 
to obtain the change in $I$. Then,

$$
\frac{\partial I}{\partial \alpha_{i}} \approx \frac{I\left(\alpha_{i}+\delta \alpha_{i}\right)-I\left(\alpha_{i}\right)}{\delta \alpha_{i}}
$$

The gradient vector $\mathcal{G}=\partial I / \partial \alpha$ may now be used to determine a direction of improvement. The simplest procedure is to make a step in the negative gradient direction by setting

$$
\boldsymbol{\alpha}^{n+1}=\boldsymbol{\alpha}^{n}+\delta \boldsymbol{\alpha}
$$

where

$$
\delta \boldsymbol{\alpha}=-\lambda \mathcal{G}
$$

so that to first order,

$$
I+\delta I=I-\mathcal{G}^{\mathrm{T}} \delta \boldsymbol{\alpha}=I-\lambda \mathcal{G}^{\mathrm{T}} \mathcal{G}<I
$$

More sophisticated search procedures may be used such as quasi-Newton methods, which attempt to estimate the second derivative $\partial^{2} I / \partial \alpha_{i} \partial \alpha_{j}$ of the cost function from changes in the gradient $\partial I / \partial \boldsymbol{\alpha}$ in successive optimization steps. The main disadvantage of this approach is the need for a number of flow calculations proportional to the number of design variables to estimate the gradient. The computational costs can thus become prohibitive as the number of design variables is increased.

In order to circumvent this difficulty, the gradient can be determined indirectly by solving an adjoint equation that has coefficients defined by the solution of the flow equations. The cost of solving the adjoint equation is comparable to that of solving the flow equations. Thus the gradient can be determined with roughly the computational costs of two flow solutions, independently of the number of design variables, which may be infinite if the boundary is regarded as a free surface. The underlying concepts are clarified by the following abstract description of the adjoint method.

For flow about an airfoil or wing, the aerodynamic properties that define the cost function are functions of the flow-field variables $w$ and the physical location of the boundary, which may be represented by the function $\mathcal{F}$, say. Then

$$
I=I(\boldsymbol{w}, \mathcal{F})
$$

and a change in $\mathcal{F}$ results in a change

$$
\delta I=\left[\frac{\partial I^{\mathrm{T}}}{\partial \boldsymbol{w}}\right] \delta \boldsymbol{w}+\left[\frac{\partial I^{\mathrm{T}}}{\partial \mathcal{F}}\right] \delta \mathcal{F}
$$

in the cost function. Suppose that the governing equation $\boldsymbol{R}$ that expresses the dependence of $\boldsymbol{w}$ and $\mathcal{F}$ within the flowfield domain $D$ can be written as

$$
\boldsymbol{R}(\boldsymbol{w}, \mathcal{F})=0
$$

Then, $\delta w$ is determined from the equation

$$
\delta \boldsymbol{R}=\left[\frac{\partial \boldsymbol{R}}{\partial \boldsymbol{w}}\right] \delta \boldsymbol{w}+\left[\frac{\partial \boldsymbol{R}}{\partial \mathcal{F}}\right] \delta \mathcal{F}=0
$$

Since the variation $\delta \boldsymbol{R}$ is zero, it can be multiplied by a Lagrange multiplier $\psi$ and subtracted from the variation $\delta I$ without changing the result. Thus, equation (9) can be replaced by

$$
\begin{aligned}
\delta I & =\frac{\partial I^{\mathrm{T}}}{\partial \boldsymbol{w}} \delta \boldsymbol{w}+\frac{\partial I^{\mathrm{T}}}{\partial \mathcal{F}} \delta \mathcal{F}-\boldsymbol{\psi}^{\mathrm{T}}\left(\left[\frac{\partial \boldsymbol{R}}{\partial \boldsymbol{w}}\right] \delta \boldsymbol{w}+\left[\frac{\partial \boldsymbol{R}}{\partial \mathcal{F}}\right] \delta \mathcal{F}\right) \\
& =\left\{\frac{\partial I^{\mathrm{T}}}{\partial \boldsymbol{w}}-\boldsymbol{\psi}^{\mathrm{T}}\left[\frac{\partial \boldsymbol{R}}{\partial \boldsymbol{w}}\right]\right\} \delta \boldsymbol{w}+\left\{\frac{\partial I^{\mathrm{T}}}{\partial \mathcal{F}}-\boldsymbol{\psi}^{\mathrm{T}}\left[\frac{\partial \boldsymbol{R}}{\partial \mathcal{F}}\right]\right\} \delta \mathcal{F}
\end{aligned}
$$

Choosing $\psi$ to satisfy the adjoint equation

$$
\left[\frac{\partial \boldsymbol{R}}{\partial \boldsymbol{w}}\right]^{\mathrm{T}} \boldsymbol{\psi}=\frac{\partial I}{\partial \boldsymbol{w}}
$$

the first term is eliminated, and we find that

$$
\delta I=\mathcal{G}^{\mathrm{T}} \delta \mathcal{F}
$$

where

$$
\mathcal{G}=\frac{\partial I^{\mathrm{T}}}{\partial \mathcal{F}}-\boldsymbol{\psi}^{\mathrm{T}}\left[\frac{\partial \boldsymbol{R}}{\partial \mathcal{F}}\right]
$$

The advantage is that (14) is independent of $\delta \boldsymbol{w}$, with the result that the gradient of $I$ with respect to an arbitrary number of design variables can be determined without the need for additional flow-field evaluations. In the case that (10) is a partial differential equation, the adjoint equation (13) is also a partial differential equation and determination of the appropriate boundary conditions requires careful mathematical treatment. In the case of the discrete adjoint method, these formulas are applied directly to the discretized cost function and flow equations. 


\section{DESIGN OPTIMIZATION PROCEDURE}

Once the gradient $\mathcal{G}$ has been determined, an improvement can be obtained by making a shape change

$$
\delta \mathcal{F}=-\lambda \mathcal{G}
$$

where $\lambda$ is sufficiently small and positive so that

$$
\delta I=-\lambda \mathcal{G}^{\mathrm{T}} \mathcal{G} \quad<0
$$

In the infinitely dimensional case where the shape is represented by a function $\mathcal{F}(x)$, the gradient is also a function $\mathcal{G}(x)$, and the increment of the cost is represented by the integral

$$
\delta I=\int \mathcal{G}(x) \delta \mathcal{F}(x) \mathrm{d} x
$$

Now, it becomes important to maintain the smoothness of the shape. For this purpose, one can set

$$
\delta \mathcal{F}=-\lambda \overline{\mathcal{G}}
$$

where $\overline{\mathcal{G}}$ satisfies the smoothing equation

$$
\overline{\mathcal{G}}-\frac{\partial}{\partial x} \epsilon \frac{\partial}{\partial x} \overline{\mathcal{G}}=\mathcal{G}, \quad \overline{\mathcal{G}}=0 \quad \text { at end points }
$$

Then

$$
\begin{aligned}
\delta I & =\int \mathcal{G}(x) \delta \mathcal{F}(x) \mathrm{d} x \\
& =-\lambda \int\left(\overline{\mathcal{G}}-\frac{\partial}{\partial x} \epsilon \frac{\partial}{\partial x} \overline{\mathcal{G}}\right) \overline{\mathcal{G}} \mathrm{d} x
\end{aligned}
$$

or, integrating by parts

$$
\delta I=-\lambda \int\left(\overline{\mathcal{G}}^{2}+\epsilon\left(\frac{\partial}{\partial x} \overline{\mathcal{G}}\right)^{2}\right) \mathrm{d} x
$$

so an improvement is still guaranteed. The smoothed gradient $\overline{\mathcal{G}}$ can be regarded as the gradient with respect to a modified Sobolev inner product

$$
(u, v)=\int\left(u v+\epsilon u^{\prime} v^{\prime}\right) \mathrm{d} x
$$

When second-order central differencing is applied to (20), the equation at a given node, $i$, can be expressed as

$$
\overline{\mathcal{G}}_{i}-\epsilon\left(\overline{\mathcal{G}}_{i+1}-2 \overline{\mathcal{G}}_{i}+\overline{\mathcal{G}}_{i-1}\right)=\mathcal{G}_{i}, \quad 1 \leq i \leq n
$$

where $\mathcal{G}_{i}$ and $\overline{\mathcal{G}}_{i}$ are the point gradients at node $i$ before and after the smoothing, respectively, and $n$ is the number of design variables equal to the number of mesh points in this case. Then,

$$
\overline{\mathcal{G}}=A \mathcal{G}
$$

where $\boldsymbol{A}$ is the $n \times n$ tridiagonal matrix such that

$$
\boldsymbol{A}^{-1}=\left[\begin{array}{ccccc}
1+2 \epsilon & -\epsilon & 0 & . & 0 \\
\epsilon & \cdot & \cdot & & \\
0 & \cdot & \cdot & \cdot & \\
\cdot & & \cdot & \cdot & -\epsilon \\
0 & & & \epsilon & 1+2 \epsilon
\end{array}\right]
$$

Then, in each design iteration, a step, $\delta \mathcal{F}$, is taken such that

$$
\delta \mathcal{F}=-\lambda A \mathcal{G}
$$

As can be seen from the form of this expression, implicit smoothing may be regarded as a preconditioner that allows the use of much larger steps for the search procedure and leads to a large reduction in the number of design iterations needed for convergence.

The design procedure can finally be summarized as follows:

1. Solve the flow equations for $\boldsymbol{w}$.

2. Solve the adjoint equations for $\psi$ subject to appropriate boundary conditions.

3. Evaluate $\mathcal{G}$ and calculate the corresponding Sobolev gradient $\overline{\mathcal{G}}$.

4. Project $\overline{\mathcal{G}}$ into an allowable subspace that satisfies any geometric constraints.

5. Update the shape based on the direction of steepest descent.

6. Return to 1 until convergence is reached.

The application of this method is illustrated for the cases of transonic potential flow and flow described by the Euler equations in the next two sections.

\section{AIRFOIL DESIGN FOR POTENTIAL FLOW USING CONFORMAL MAPPING}

Consider the case of two-dimensional compressible inviscid flow. In the absence of shock waves, an initially irrotational flow will remain irrotational, and we can assume that the velocity vector $\boldsymbol{q}$ is the gradient of a potential $\phi$. In the 
presence of weak shock waves this remains a fairly good approximation. Let $p, \rho, c$, and $M$ be the pressure, density, speed of sound, and Mach number $q / c$. Then, the potential flow equation is

$$
\nabla \cdot(\rho \nabla \phi)=0
$$

where the density is given by

$$
\rho=\left[1+\frac{\gamma-1}{2} M_{\infty}^{2}\left(1-q^{2}\right)\right]^{\frac{1}{\gamma-1}}
$$

while

$$
p=\frac{\rho^{\gamma}}{\gamma M_{\infty}^{2}}, \quad c^{2}=\frac{\gamma p}{\rho}
$$

Here, $M_{\infty}$ is the Mach number in the freestream, and the units have been chosen so that $p$ and $q$ have a value of unity in the far field.

Suppose that the domain $D$ exterior to the airfoil profile $C$ in the $z$ plane is conformally mapped on to the domain exterior to a unit circle in the $\sigma$ plane as sketched in Figure 3. Let $R$ and $\theta$ be polar coordinates in the $\sigma$ plane, and let $r$ be the inverted radial coordinate $1 / R$. Also, let $h$ be the modulus of the derivative of the mapping function

$$
h=\left|\frac{\mathrm{d} z}{\mathrm{~d} \sigma}\right|
$$

Now the potential flow equation becomes

$$
\frac{\partial}{\partial \theta}\left(\rho \phi_{\theta}\right)+r \frac{\partial}{\partial r}\left(r \rho \phi_{r}\right)=0 \text { in } D
$$

where the density is given by equation (29), and the circumferential and radial velocity components are

$$
u=\frac{r \phi_{\theta}}{h}, \quad v=\frac{r^{2} \phi_{r}}{h}
$$

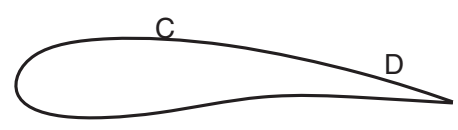

(a)

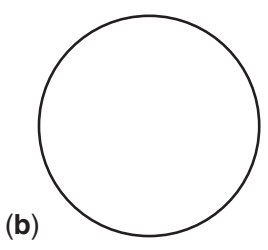

(b)

Figure 3. Conformal mapping. (a) $z$ plane; (b) $\sigma$ plane. while

$$
q^{2}=u^{2}+v^{2}
$$

The condition of flow tangency leads to the Neumann boundary condition

$$
v=\frac{1}{h} \frac{\partial \phi}{\partial r}=0 \text { on } C
$$

In the far field, the potential is given by an asymptotic estimate, leading to a Dirichlet boundary condition at $r=0$.

Suppose that it is desired to achieve a specified velocity distribution $q_{\mathrm{d}}$ on $C$. Introduce the cost function

$$
I=\frac{1}{2} \int_{C}\left(q-q_{\mathrm{d}}\right)^{2} \mathrm{~d} \theta
$$

The design problem is now treated as a control problem where the control function is the mapping modulus $h$, which is to be chosen to minimize $I$ subject to the constraints defined by the flow equations (28-35).

A modification $\delta h$ to the mapping modulus will result in variations $\delta \phi, \delta u, \delta v$, and $\delta \rho$ to the potential, velocity components, and density. The resulting variation in the cost will be

$$
\delta I=\int_{C}\left(q-q_{\mathrm{d}}\right) \delta q \mathrm{~d} \theta
$$

where, on $C, q=u$. Also,

$$
\delta u=r \frac{\delta \phi_{\theta}}{h}-u \frac{\delta h}{h}, \quad \delta v=r^{2} \frac{\delta \phi_{r}}{h}-v \frac{\delta h}{h}
$$

while according to equation (29)

$$
\frac{\partial \rho}{\partial u}=-\frac{\rho u}{c^{2}}, \quad \frac{\partial \rho}{\partial v}=-\frac{\rho v}{c^{2}}
$$

It follows that $\delta \phi$ satisfies

$$
L \delta \phi=-\frac{\partial}{\partial \theta}\left(\rho M^{2} \phi_{\theta} \frac{\delta h}{h}\right)-r \frac{\partial}{\partial r}\left(\rho M^{2} r \phi_{r} \frac{\delta h}{h}\right)
$$

where

$$
\begin{aligned}
L \equiv & \frac{\partial}{\partial \theta}\left[\rho\left(1-\frac{u^{2}}{c^{2}}\right) \frac{\partial}{\partial \theta}-\frac{\rho u v}{c^{2}} r \frac{\partial}{\partial r}\right] \\
& +r \frac{\partial}{\partial r}\left[\rho\left(1-\frac{v^{2}}{c^{2}}\right) r \frac{\partial}{\partial r}-\frac{\rho u v}{c^{2}} \frac{\partial}{\partial \theta}\right]
\end{aligned}
$$


Then, if $\psi$ is any periodic differentiable function that vanishes in the far field,

$$
\int_{D} \frac{\psi}{r^{2}} L \delta \phi \mathrm{d} S=\int_{D} \rho M^{2} \nabla \phi \cdot \nabla \psi \frac{\delta h}{h} \mathrm{~d} S
$$

where $\mathrm{d} S$ is the area element $r \mathrm{~d} r \mathrm{~d} \theta$, and the right-hand side has been integrated by parts.

Now, we can augment equation (37) by subtracting the constraint (42). The auxiliary function $\psi$ then plays the role of a Lagrange multiplier. Thus,

$$
\begin{aligned}
\delta I= & \int_{C}\left(q-q_{\mathrm{d}}\right) q \frac{\delta h}{h} \mathrm{~d} \theta-\int_{C} \delta \phi \frac{\partial}{\partial \theta}\left(\frac{q-q_{\mathrm{d}}}{h}\right) \mathrm{d} \theta \\
& -\int_{D} \frac{\psi}{r^{2}} L \delta \phi \mathrm{d} S+\int_{D} \rho M^{2} \nabla \phi \cdot \nabla \psi \frac{\delta h}{h} \mathrm{~d} S
\end{aligned}
$$

Now suppose that $\psi$ satisfies the adjoint equation

$$
L \psi=0 \text { in } D
$$

with the boundary condition

$$
\frac{\partial \psi}{\partial r}=\frac{1}{\rho} \frac{\partial}{\partial \theta}\left(\frac{q-q_{\mathrm{d}}}{h}\right) \text { on } C
$$

Then, integrating by parts,

$$
\delta I=-\int_{C}\left(q-q_{\mathrm{d}}\right) q \frac{\delta h}{h} \mathrm{~d} \theta+\int_{D} \rho M^{2} \nabla \phi \cdot \nabla \psi \frac{\delta h}{h} \mathrm{~d} S
$$

Here, the first term represents the direct effect of the change in the metric, while the area integral represents a correction for the effect of compressibility. When the second term is deleted, the method reduces to a variation of Lighthill's method (Lighthill, 1945).

Equation (46) can be further simplified to represent $\delta I$ purely as a boundary integral because the mapping function is fully determined by the value of its modulus on the boundary. Set

$$
\log \frac{\mathrm{d} z}{\mathrm{~d} \sigma}=\mathcal{F}+i \beta
$$

where

$$
\mathcal{F}=\log \left|\frac{\mathrm{d} z}{\mathrm{~d} \sigma}\right|=\log h
$$

and

$$
\delta \mathcal{F}=\frac{\delta h}{h}
$$

Then, $\mathcal{F}$ satisfies Laplace's equation

$$
\Delta \mathcal{F}=0 \text { in } D
$$

and if there is no stretching in the far field, $\mathcal{F} \rightarrow 0$. Introduce another auxiliary function $P$ which satisfies

$$
\Delta P=\rho M^{2} \nabla \psi \cdot \nabla \psi \text { in } D
$$

and

$$
P=0 \text { on } C
$$

Then, after integrating by parts, we find that

$$
\delta I=\int_{C} \mathcal{G} \delta \mathcal{F}_{c} \mathrm{~d} \theta
$$

where $\mathcal{F}_{\mathrm{c}}$ is the boundary value of $\mathcal{F}$, and

$$
\mathcal{G}=\frac{\partial P}{\partial r}-\left(q-q_{\mathrm{d}}\right) q
$$

Thus, we can attain an improvement by a modification

$$
\delta \mathcal{F}_{\mathrm{c}}=-\lambda \overline{\mathcal{G}}
$$

in the modulus of the mapping function on the boundary, which in turn defines the computed mapping function since $\mathcal{F}$ satisfies Laplace's equation. In this way, the Lighthill method is extended to transonic flow. More details can be found in the studies by Jameson (1988) and Jameson and Martinelli (1999).

\section{WING DESIGN USING THE EULER EQUATIONS}

The application of control theory to aerodynamic design problems is illustrated in this section for the case of threedimensional wing design using the compressible Euler equations as the mathematical model. The extension of the method to treat the Navier-Stokes equations is presented in references Jameson, Martinelli and Pierce (1998), Jameson and Martinelli (1999), and Jameson (2003). It proves convenient to denote the Cartesian coordinates and velocity 
components by $x_{1}, x_{2}, x_{3}$ and $u_{1}, u_{2}, u_{3}$ and to use the convention that summation over $i=1$ to 3 is implied by a repeated index $i$. Then, the three-dimensional Euler equations may be written as

$$
\frac{\partial \boldsymbol{w}}{\partial t}+\frac{\partial \boldsymbol{f}_{i}}{\partial x_{i}}=0
$$

where

$$
\boldsymbol{w}=\left[\begin{array}{c}
\rho \\
\rho u_{1} \\
\rho u_{2} \\
\rho u_{3} \\
\rho E
\end{array}\right], \quad \boldsymbol{f}_{i}=\left[\begin{array}{c}
\rho u_{i} \\
\rho u_{i} u_{1}+p \delta_{i 1} \\
\rho u_{i} u_{2}+p \delta_{i 2} \\
\rho u_{i} u_{3}+p \delta_{i 3} \\
\rho u_{i} H
\end{array}\right]
$$

and $\delta_{i j}$ is the Kronecker delta function. Also

$$
p=(\gamma-1) \rho\left(E-\frac{1}{2} u_{i}^{2}\right)
$$

and

$$
\rho H=\rho E+p
$$

where $\gamma$ is the ratio of the specific heats.

In order to simplify the derivation of the adjoint equations, we map the solution to a fixed computational domain $D$ with coordinates $\xi_{1}, \xi_{2}, \xi_{3}$ where

$$
K_{i j}=\left[\frac{\partial x_{i}}{\partial \xi_{j}}\right], \quad J=\operatorname{det}(\boldsymbol{K}), \quad K_{i j}^{-1}=\left[\frac{\partial \xi_{i}}{\partial x_{j}}\right]
$$

and

$$
\boldsymbol{S}=J \boldsymbol{K}^{-1}
$$

The elements of $\boldsymbol{S}$ are the cofactors of $\boldsymbol{K}$, and in a finite volume discretization they are just the face areas of the computational cells projected in the $x_{1}, x_{2}$, and $x_{3}$ directions, while $J$ represents the cell volume.

Now, multiplying equation(56) by $J$ and applying the chain rule,

$$
J \frac{\partial \boldsymbol{w}}{\partial t}+\boldsymbol{R}(\boldsymbol{w})=0
$$

where

$$
\boldsymbol{R}(\boldsymbol{w})=S_{i j} \frac{\partial \boldsymbol{f}_{j}}{\partial \xi_{i}}=\frac{\partial}{\partial \xi_{i}}\left(S_{i j} \boldsymbol{f}_{j}\right)
$$

We can write the transformed fluxes in terms of the scaled contravariant velocity components

$$
U_{i}=S_{i j} u_{j}
$$

as

$$
\boldsymbol{F}_{i}=S_{i j} f_{j}=\left[\begin{array}{c}
\rho U_{i} \\
\rho U_{i} u_{1}+S_{i 1} p \\
\rho U_{i} u_{2}+S_{i 2} p \\
\rho U_{i} u_{3}+S_{i 3} p \\
\rho U_{i} H
\end{array}\right]
$$

For convenience, the coordinates $\xi_{i}$ describing the fixed computational domain are chosen so that each boundary conforms to a constant value of one of these coordinates. Variations in the shape then result in corresponding variations in the mapping derivatives defined by $S_{i j}$. Moreover, it will be assumed that the portion of the boundary that undergoes shape modifications is restricted to the coordinate surface $\xi_{2}=0$.

In order to illustrate the procedure, consider an inverse problem where we aim to attain a desired surface pressure $p_{\mathrm{d}}$. Then, a natural choice is to define the cost function as

$$
I=\frac{1}{2} \int_{B}\left(p-p_{\mathrm{d}}\right)^{2} \mathrm{~d} S
$$

where $p_{\mathrm{d}}$ is the desired surface pressure, and the integral is evaluated over the actual surface area of the wing or any other shape that is to be redesigned. In the computational domain this is transformed to

$$
I=\frac{1}{2} \iint_{B}\left(p-p_{\mathrm{d}}\right)^{2}\left|\boldsymbol{S}_{2}\right| \mathrm{d} \xi_{1} \mathrm{~d} \xi_{3}
$$

where the quantity

$$
\left|\boldsymbol{S}_{2}\right|=\sqrt{S_{2 j} S_{2 j}}
$$

denotes the face area corresponding to a unit element of face area in the computational domain.

In the steady state, it follows from equation (63) that the variation $\delta \boldsymbol{w}$ in the solution due to a shape change is determined by

$$
\delta \boldsymbol{R}=\frac{\partial}{\partial \xi_{i}} \delta \boldsymbol{F}_{i}=0
$$

where

$$
\delta \boldsymbol{F}_{i}=S_{i j} \frac{\partial \boldsymbol{F}_{j}}{\partial \boldsymbol{w}} \delta \boldsymbol{w}+\delta S_{i j} \boldsymbol{f}_{j}
$$


Here, the shape change appears in the metric variations $\delta S_{i j}$. Now we multiply this by a Lagrange multiplier $\boldsymbol{\psi}^{\mathrm{T}}$, generally called a costate vector, and subtract its integral over the flow domain from the variation in the cost to obtain

$$
\begin{aligned}
\delta I= & \iint_{B}\left(p-p_{\mathrm{d}}\right) \delta p|\boldsymbol{S}| \mathrm{d} \xi_{1} \xi_{3} \\
& +\frac{1}{2} \iint_{B}\left(p-p_{\mathrm{d}}\right)^{2}|\delta \boldsymbol{S}|_{2} \mathrm{~d} \xi_{1} \mathrm{~d} \xi_{3}-\int_{D} \boldsymbol{\psi}^{\mathrm{T}} \frac{\partial}{\partial \xi_{i}} \delta \boldsymbol{F}_{i} \mathrm{~d} \xi
\end{aligned}
$$

Next, the last term may be integrated by parts to give

$$
\begin{aligned}
& -\iint_{B} \boldsymbol{\psi}^{\mathrm{T}} \delta \boldsymbol{F}_{2} \mathrm{~d} \xi_{1} \mathrm{~d} \xi_{3}-\int \frac{\partial \boldsymbol{\psi}^{\mathrm{T}}}{\partial \xi}\left(S_{i j} \frac{\partial \boldsymbol{f}_{j}}{\partial \boldsymbol{w}}\right) \delta \boldsymbol{w} \mathrm{d} \xi \\
& -\int_{D} \frac{\partial \boldsymbol{\psi}^{\mathrm{T}}}{\partial \xi} \delta S_{i j} \boldsymbol{f}_{j}(\boldsymbol{w}) \mathrm{d} \xi
\end{aligned}
$$

where contributions from the far-field boundaries have been eliminated by appropriate choice of the far-field adjoint boundary conditions (Jameson (1988)).

Now it can be seen that any explicit dependence of the cost variation $\delta I$ on $\delta w$ can be eliminated by requiring the costate vector $\psi$ to satisfy the adjoint equation

$$
\left[S_{i j} \frac{\partial \boldsymbol{f}}{\partial \boldsymbol{w}}\right]^{\mathrm{T}} \frac{\partial \boldsymbol{\psi}}{\partial \xi_{i}}=0 \text { in } D
$$

and also canceling the first boundary integral containing $\delta p$ by the boundary integral containing $\delta \boldsymbol{F}_{2}$. Now the condition that there is no flow through the wall boundary at $\xi_{2}=0$ is equivalent to

$$
U_{2}=0
$$

so that

$$
\delta U_{2}=0
$$

when the boundary shape is modified. Consequently, the variation of the inviscid flux at the boundary reduces to

$$
\delta \boldsymbol{F}_{2}=\delta p\left[\begin{array}{c}
0 \\
S_{21} \\
S_{22} \\
S_{23} \\
0
\end{array}\right]+p\left[\begin{array}{c}
0 \\
\delta S_{21} \\
\delta S_{22} \\
\delta S_{23} \\
0
\end{array}\right]
$$

Accordingly, the desired cancellation is achieved by requiring $\psi$ to satisfy the boundary condition

$$
\psi_{j} n_{j}=p-p_{\mathrm{d}}
$$

where $n_{j}$ are the components of the surface normal. The variation in the cost is thus reduced to

$$
\begin{aligned}
\delta I= & \frac{1}{2} \iint_{B}\left(p-p_{\mathrm{d}}\right)^{2}|\delta \mathbf{S}|_{2} \mathrm{~d} \xi_{1} \mathrm{~d} \xi_{3}-\iint_{B}\left(\delta S_{21} \psi_{2}+\delta S_{22} \psi_{3}\right. \\
& \left.+\delta S_{23} \psi_{4}\right) p \mathrm{~d} \xi_{1} \mathrm{~d} \xi_{3}+\int_{D} \frac{\partial \boldsymbol{\psi}^{\mathrm{T}}}{\partial \xi} \delta S_{i j} \boldsymbol{f}_{j}(\boldsymbol{w}) \mathrm{d} \xi
\end{aligned}
$$

Finally, an explicit formulation for the gradient can be extracted by defining the way in which the mesh is deformed when the shape is modified and hence the metric perturbations $\delta S_{i j}$. Other cost functions can be addressed by appropriate modification of the adjoint boundary condition to cancel the boundary integral in the cost variation. Since $\delta \boldsymbol{F}_{2}$ depends only on the pressure variation $\delta p$ and the metric variations, any cost function involving an integral of the surface pressure can be treated. Some representative examples are presented in the following section.

\section{DESIGN CASE STUDIES}

\subsection{Two-dimensional studies of transonic airfoil design}

When the inviscid Euler equations are used to model the flow, the source of drag is the wave drag due to shock waves. Accordingly, if the shape is optimized for minimum drag at fixed lift, the best attainable result is a shock-free airfoil with zero drag. By this criterion, the optimum shape is completely non-unique, since all shock-free profiles are equally good. Experience during the last 15 years has confirmed that shock-free profiles can be obtained from a wide variety of initial shape, while maintaining a fixed lift coefficient and a fixed thickness. An example of shock-free design is shown in Figure 4 for the optimization of a DLBA-243 airfoil (Harbeck and Jameson, 2005).

\subsection{Shark Reno Racer design}

Using three-dimensional optimization, it is possible to arrive very rapidly at an efficient design. This is illustrated in the next example that is the wing design for a proposed 
(a)
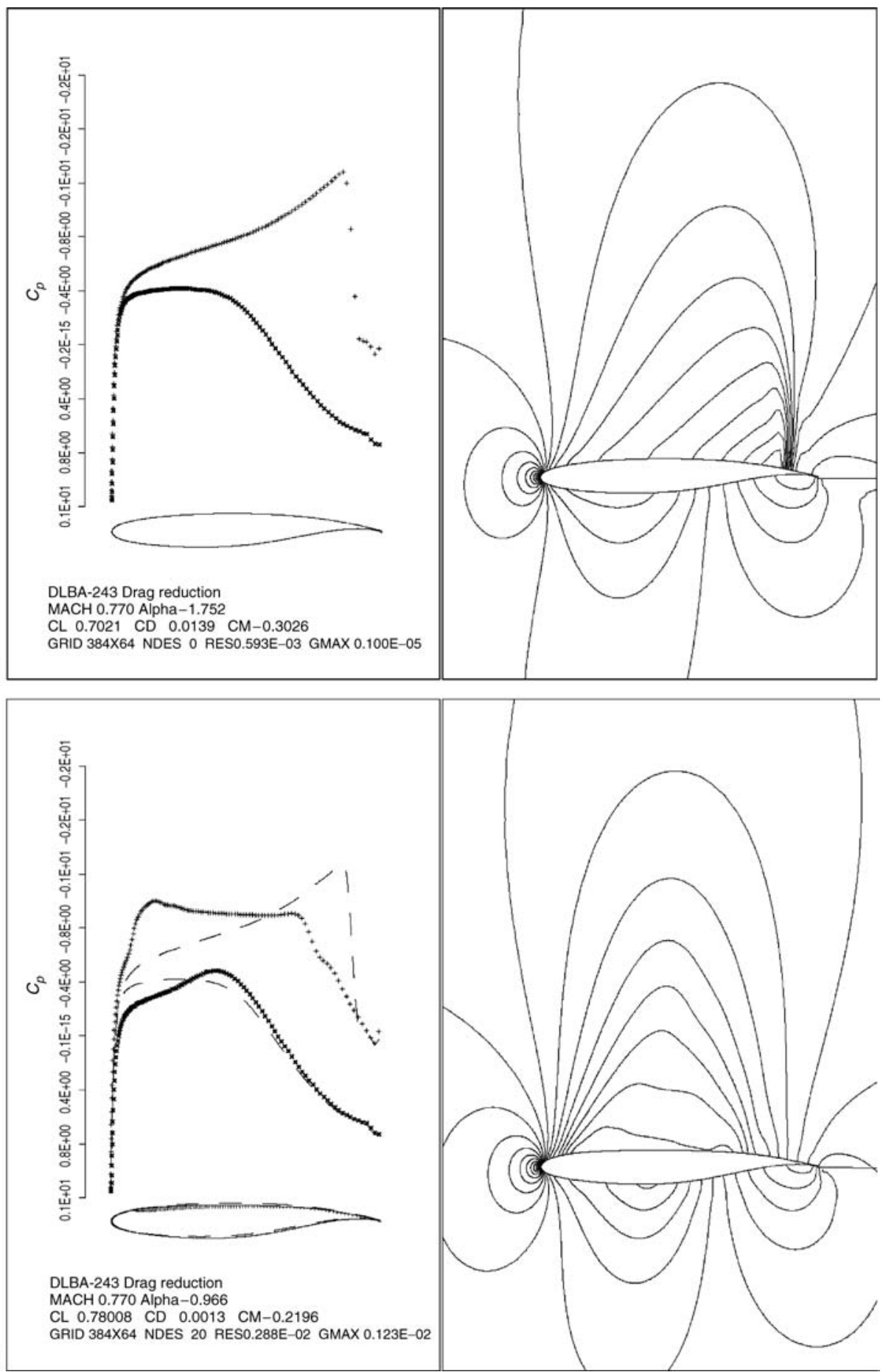

Figure 4. Pressure distribution and Mach contours for the DLBA-243 airfoil. (a) Before optimization; (b) after optimization.

propeller-driven Reno Air Racer, the Shark, which was intended to reach speeds of $550 \mathrm{mph}$ (Jameson and Vassberg, 2001). A sketch of Shark is shown in Figure 5a.

The initial wing had extremely strong shock waves that were removed by optimization using the Euler equations, as shown in Figure $5 b$ and $5 c$. Then the design was further refined by inverse design with the Reynolds-averaged Navier-Stokes equations to produce a pressure distribution that could promote laminar flow over a range of lift coefficients as shown in Figure 5d. 

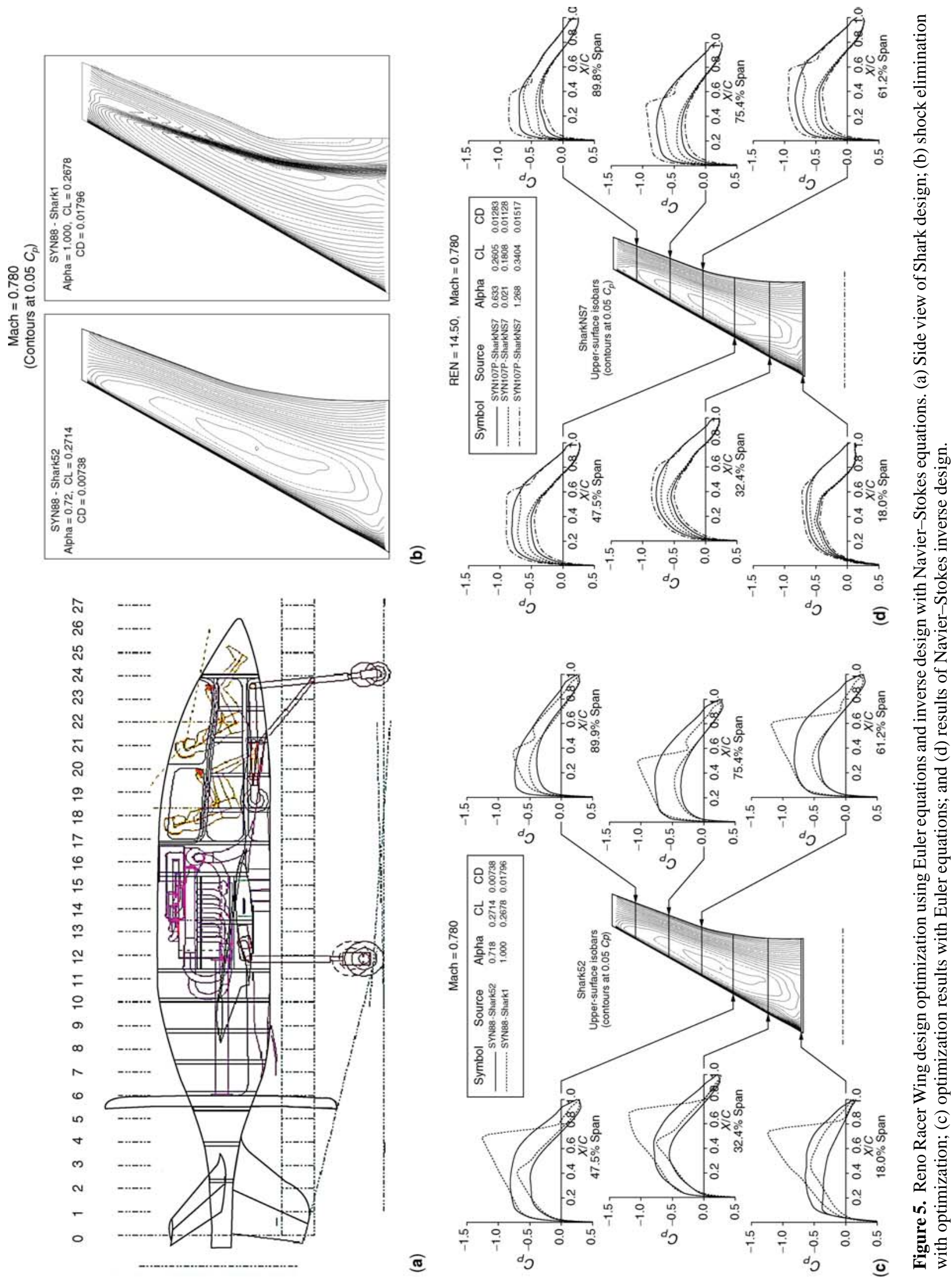

은
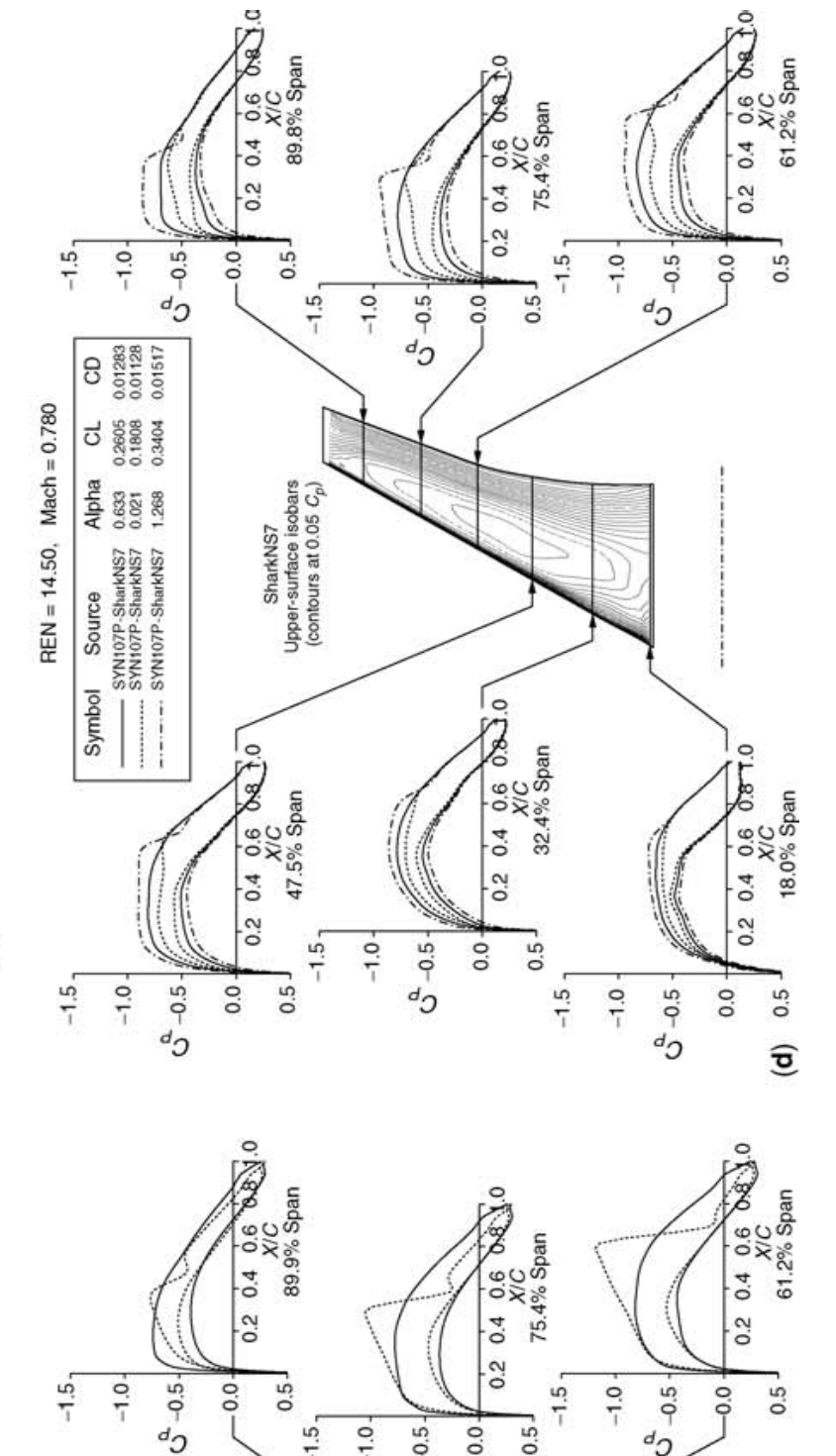

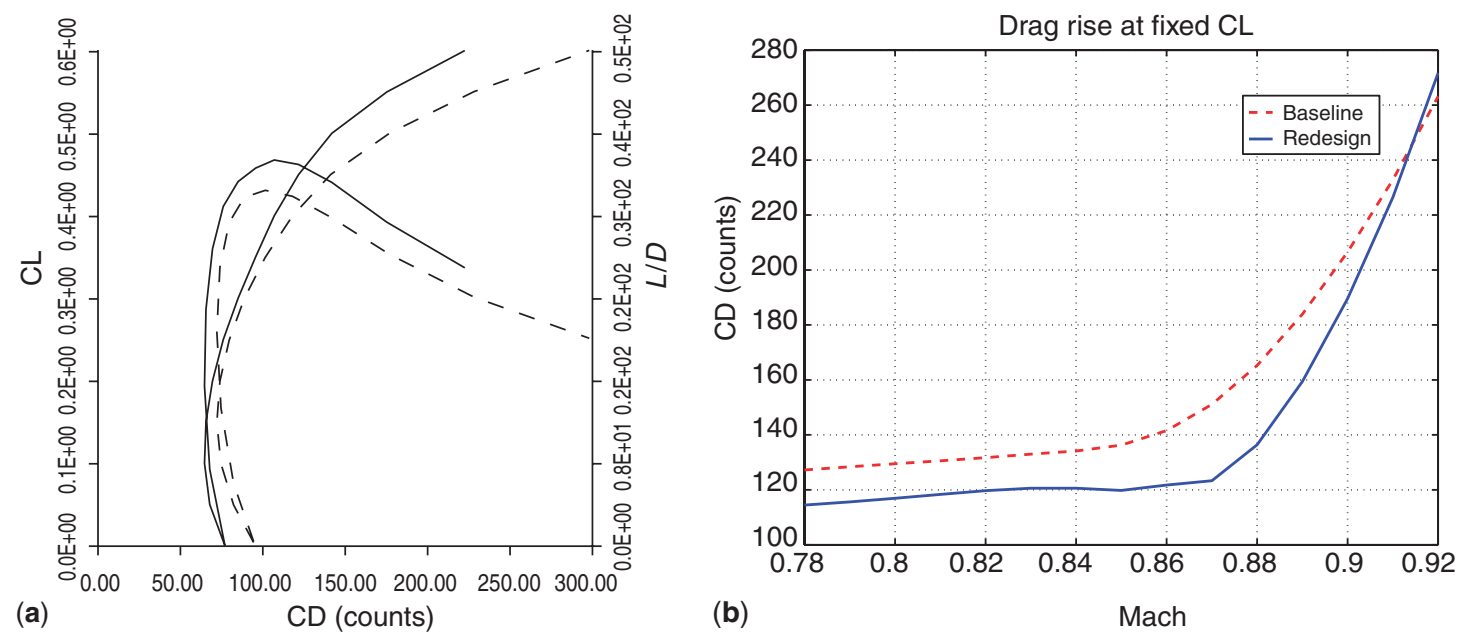

Figure 6. (a) Drag Polars of Baseline and Super B747 at Mach 0.86; (b) Drag vs. Mach no. of Super B747. Solid line represents Super B747. Dash line represents Baseline B747.

\subsection{Super B747}

In order to obtain the maximum possible benefit, one should allow for redesign of the planform as well as the wing section shapes. Then, it is necessary to estimate the change in the wing weight that will result from the redesign. The next example, a new wing design for a "Super Boeing 747", with an increased span, reduced sweep, and a much thicker wing section to reduce the weight, illustrates the kind of improvements that can be obtained by optimization. The numbers in Figure 6a show, while the curve in Figure 6b compares the drag rise of the "Super B747" with the existing B747 (Leoviriyakit and Jameson, 2004).

\subsection{Shape optimization of a transonic business jet}

The same design method has also been applied to several complete aircraft configurations using unstructured meshes (Jameson, Shankaran and Martinelli, 2008). The results for a business jet are shown in Figure 7a and $7 b$. There is a strong shock over the outboard wing sections of the initial configuration, which is essentially eliminated by the redesign. The drag was reduced from 235 counts to 215 counts in about eight design cycles. The lift was constrained at 0.4 by perturbing the angle of attack. Further, the original thickness of the wing was maintained during the design process ensuring that fuel volume and structural integrity will be maintained by the redesigned shape. Thickness constraints on the wing were imposed on cutting planes along the span of the wing and by transferring the constrained shape movement back to the nodes of the surface triangulation.

\section{CONCLUSION}

The adjoint design method presented in these notes is now well established and has proved effective in a variety of industrial applications including, most recently, the wing design of the Gulfstream G650. The method combines the versatility of numerical optimization methods with the efficiency of inverse design methods. The geometry is modified by a (a)

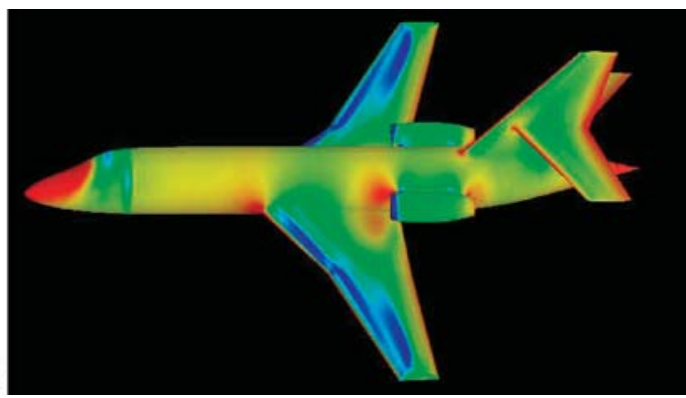

(b)

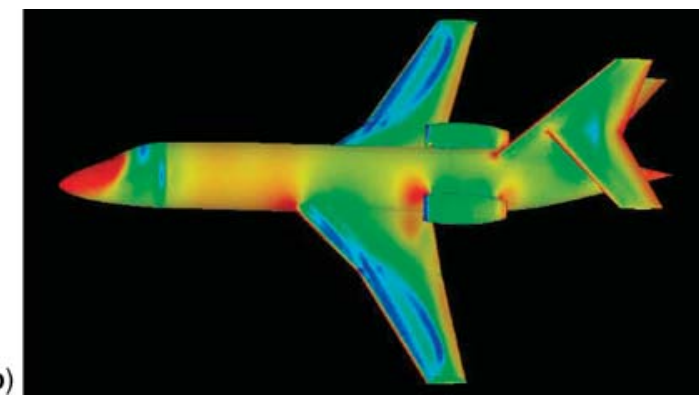

Figure 7. Density contours for a business jet at $M=0.8, \alpha=2^{\circ}$. (a) Baseline; (b) Redesign. 
grid perturbation technique, which is applicable to arbitrary configurations. The combination of computational efficiency with geometric flexibility provides a powerful tool, with the final goal being to create practical aerodynamic shape design methods for complete aircraft configurations. Motivated by the need to optimize complete configurations, the further development of adjoint methods on unstructured meshes is a subject of ongoing research (Nielsen and Park, 2006; Castro and Zuazua, 2007; Mavriplis, 2007). It may eventually prove most effective to incorporate CAD definitions of the geometry directly into the shape modification procedure.

\section{ACKNOWLEDGMENT}

The author's research on shape optimization has benefited greatly from the continuing support over more than 20 years of the Air Force Office of Scientific Research through its Computational Mathematics Program.

\section{REFERENCES}

Anderson, W.K. and Venkatakrishnan, V. (1997) Aerodynamic design and optimization on unstructured grids with a continuous adjoint formulation. AIAA 35th Aerospace Sciences Meeting, Reno. AIAA Paper 97-0643.

Baysal, O. and Eleshaky, M.E. (1991) Aerodynamic design optimization using sensitivity analysis and computational fluid dynamics. 29th Aerospace Sciences Meeting, Reno. AIAA Paper 91-0471.

Cabuk,H., Shung, C.H. and Modi, V. (1991) Adjoint operator approach to shape design for internal incompressible flow. Third International Conference on Inverse Design Concepts and Optimization in Engineering Sciences, Pennsylvania State University, pp. 391-404.

Castro, C. and Zuazua, E. (2007) Systematic continuous adjoint approach to viscous aerodynamic design on unstructured grids. AIAA J., 45(9), 2125-2139.

Desai, M. and Ito, K. (1994) Optimal controls of Navier-Stokes equations. SIAM J. Control Optim., 32(5), 1428-1446.

Elliot, J. and Peraire, J. (1996) Practical 3D aerodynamic design and optimization using unstructured meshes. 6th AIAA/NASA/ USAF Multidisciplinary and Optimization Symposium, Seattle. AIAA Paper 96-4710.

Epstein, B. and Peigin, S. (2005) Constrained aerodynamic optimization of three-dimensional wings driven by Navier-Stokes computations. AIAA J., 43, 1946-1957.

Glauert, H. (1926) The Elements of Aerofoil and Airscrew Theory, Cambridge University Press.

Gonzalez, L.F., Whitney, E.J., Srinivas, K., and Periaux, J. (2004) Multidisciplinary aircraft design and optimisation using a robust evolutionary technique with variable fidelity models. 10th AIAA/ISSMO Multidisciplinary Analysis and Optimization Conference, Albany, NY. AIAA Paper 2004-4625.
Harbeck, M. and Jameson, A. (2005) Exploring the limits of transonic shock-free airfoil design. 43rd AIAA Aerospace Sciences Meeting \& Exhibit, Reno, NV. AIAA Paper 2005-1041.

Hicks, R.M., Murman, E.M. and Vanderplaats, G.N. (1974) An assessment of airfoil design by numerical optimization. NASA TM, X-3092.

Hicks, R.M. and Henne, P.A. (1979) Wing design by numerical optimization. AIAA 17th Aerospace Sciences Meeting, New Orleans. AIAA Paper 79-0080.

Huan, J.C. and Modi, V. (1994) Optimum design for drag minimizing bodies in incompressible flow. Inverse Prob. Eng. 1, $1-25$.

Jameson, A. (1988) Aerodynamic design via control theory. J. Sci. Comput., 3, 233-260.

Jameson, A. (1989) Computational aerodynamics for aircraft design. Science, 245, 361-371.

Jameson, A. (1995) Optimum aerodynamic design using CFD and control theory. 12th AIAA Computational Fluid Dynamics Conference, San Diego. AIAA Paper 95-1729.

Jameson, A., Martinelli, L., and Pierce, N. (1998) Optimum aerodynamic design using the Navier-Stokes equations. Theor. Comput. Fluid Dyn., 10, 213-237.

Jameson, A. and Martinelli, L. (1999) Aerodynamic shape optimization techniques based on control theory. Centro Internazionale Matematico Estivo (C.I.M.E.), Martina Franca, Italy. 151-222.

Jameson, A. and Vassberg, J.C. (2001) Computational fluid dynamics for aerodynamic design: its current and future impact. 39th AIAA Aerospace Sciences Meeting and Exhibit, Reno, NV. AIAA Paper 2001-0538.

Jameson, A. (2003) Aerodynamic shape optimization using the adjoint method. 2002-2003 Lecture Series at the Von Karman Institute for Fluid Dynamics, Brussel, Belgium.

Jameson, A., Shankaran, S. and Martinelli, L. (2008) A continuous adjoint method for unstructured grids. 16th AIAA Computational Fluid Dynamic Conference, Orlando, FL. AIAA Paper 2003-3955; AIAA J. 46(5), 1226-1239.

Jameson, A. (2004) Efficient aerodynamic shape optimization. 10th AIAA/ISSMO Multidisciplany Analysis and Optimization Conference, Albany, New York. AIAA Paper 2004-4369.

Jones, R.T. (1981) The minimum drag of thin wings in frictionless flow. J. Aerosol Sci., 18, 75-81.

LeDoux, S.T., Herling, W.W., Fatta, J. and Ratcliff, R.R. (2004) Multidisciplinary design and optimization system using higher order analysis code. 10th AIAA/ISSMO Multidisciplinary Analysis and Optimization Conference, Albany, NY. AIAA Paper 2004-4567.

Leoviriyakit, K. and Jameson, A. (2004) Case studies in aerostructural wing planform and section optimization. 22nd AIAA Applied Aerodynamics Conference and Exhibit, Rhode Island. AIAA Paper 2004-5372.

Lighthill, M.J. (1945) A new method of two dimensional aerodynamic design. Rep. Memor. Aero. Res. Coun. Lond., 2112, 143-236.

Lions, J.L. (1971) Optimal Control of Systems Governed by Partial Differential Equations, Springer-Verlag, New York. 


\section{Computational Fluid Dynamics}

Mavriplis, D.J. (2007) A discrete adjoint based approach for optimization problems on three-dimensional unstructured meshes. AIAA J., 45(44), 740-750.

Nielsen, E.J. and Anderson, W.K. (1999) Aerodynamic design optimization on unstructured meshes using the Navier-Stokes equations. AIAA J., 37(11), 1411-1419.

Nielsen, E.J. and Park, M.A. (2006) Using an adjoint approach to eliminate mesh sensitivities in computational design. AIAA J., $\mathbf{4 4}$, 948-953.

Pironneau, O. (1984) Optimal Shape Design for Elliptic Systems, Springer-Verlag, New York.
Prandtl, L. and Tietjens, O.G. (1934) Applied Hydro and Aerodynamics, Dover Publications.

Reuther, J. and Jameson, A. (1995) Aerodynamic shape optimization of wing and wing-body configurations using control theory. AIAA 33rd Aerospace Sciences Meeting and Exhibit, Reno. AIAA Paper 95-0213.

Sears, W.D. (1947) On projectiles of minimum drag. Q. Appl. Math., 4, 361-366.

Ta'asan, S., Kuruvila, G. and Salas, M.D. (1992) Aerodynamic design and optimization in one shot. 30th Aerospace Sciences Meeting and Exibit, Reno, NV. AIAA Paper 92-005. 